\title{
First observations of Pulsars with the DArk Matter Particle Explorer
}

\author{
Maria Muñoz Salinas* \\ University Geneva \\ E-mail: maria.munozeunige.ch
}

\section{Xin Wu, Stephan Zimmer}

University of Geneva

\section{Fabio Gargano}

INFN Bari

\section{Zhaoqiang Shen}

Purple Mountain Observatory

on behalf of the DAMPE Collaboration

The DArk Matter Particle Explorer (DAMPE) is a satellite-borne particle detector used to study High Energy Cosmic Rays and High Energy Gamma-Rays. It was successfully launched on the 17th of December 2015 from the Jiuquan Launching Center into a sun-synchronous orbit at an altitude of $500 \mathrm{~km}$. The instrument consists of a BGO Electromagnetic Calorimeter for electron/proton separation; a silicon tracker inter-spaced with tungsten for pair conversion of Gamma-Rays; a plastic scintillator detector which is used both as an anti-coincidence detector and for charge measurements and a neutron detector. During the first year of the mission, DAMPE has scanned the full sky at least once, thereby revealing several bright gamma-ray sources. We present an initial analysis on the brightest pulsars observed in an energy range from $1 \mathrm{GeV}$ to $100 \mathrm{GeV}$ together with an outlook on future developments. Comparisons with measurements performed by other instruments will be presented.

35th International Cosmic Ray Conference

10-20 July, 2017

Bexco, Busan, Korea

\footnotetext{
* Speaker.
} 


\section{The DArk Matter Particle Explore}

The DArk Matter Particle Explorer (DAMPE) was launched the 17th of December 2015. Since then it has been scanning the gamma-ray sky while orbiting in a sun-synchronous orbit (SSO) at an altitude of $500 \mathrm{~km}$. DAMPE consists of four sub-detectors, from bottom to top these are:

- Neutron Detector (NUD) consisting of four blocks of boron-loaded plastics scintillators wrapped in aluminium film, used for hadron rejection for energies above $150 \mathrm{GeV}$.

- Bismuth Germanium Oxide (BGO) calorimeter that comprises 308 BGO scintillating bars, arranged in 14 layers. Each bar has two PMT's at both ends of the bars for read out. Allowing the measurement of the shower profile, therefore vital for the electron/proton separation.

- Silicon tungsten tracker (STK) is composed of six double-layers of silicon strips, for resolving the incoming direction of the high energy particles. The first three layers have a tungsten sheet interlayer with a thickness of $1 \mathrm{~mm}$ in order to increase the pair conversion cross section.

- Plastic Scintillator Detector (PSD) is a double layer array of plastic strips wich covers the full entrace of the instrument. They are designed for charge measurements and to form an anti-coincidence detector for the electron/photon separation. [1]

DAMPE's main scientific objectives include the detection and study of gamma-rays from galactic and extra-galactic origin and to study the emitting sources. Here we present a first look into the gamma-ray analysis, focusing on the analysis of pulsars observed with DAMPE during the first eighteen months of operation in the survey-mode.

\subsection{Gamma-rays}

Over the last 30 years there has been a remarkable progress in the study of gamma-ray sources from both ground-based and space-based experiments. With the objective of untangling and understanding the nature and emission mechanisms of these different sources which range from large sources such as active galactic nuclei (AGN) to objects of the kilometer scale such as neutron stars. We applied a gamma-ray selection by using DAMPE's properties. Using shower profile cuts and neutrality on the PSD for proton and electron rejection respectively. We aim to reconstruct gammas that convert after the first tungsten layer in the STK.

For the study of pulsars we applied a photon selection with the objective to detect these bright sources as well as using their pulsed emission to study our signal to background as well as to optimize the selection itself. The selection of the data for this paper consist on the low energy analysis with photons from $1 \mathrm{GeV}$ to $100 \mathrm{GeV}$. The data covers the first 18 months of operations, starting from the 1st January 2016. The first days of data are excluded as during this period calibration and performance runs were being performed. The selection consist on a gamma reconstruction that searches to maximises our effective area and acceptance. We reject events obtained while the satellite crosses the South Atlantic Anomaly. 


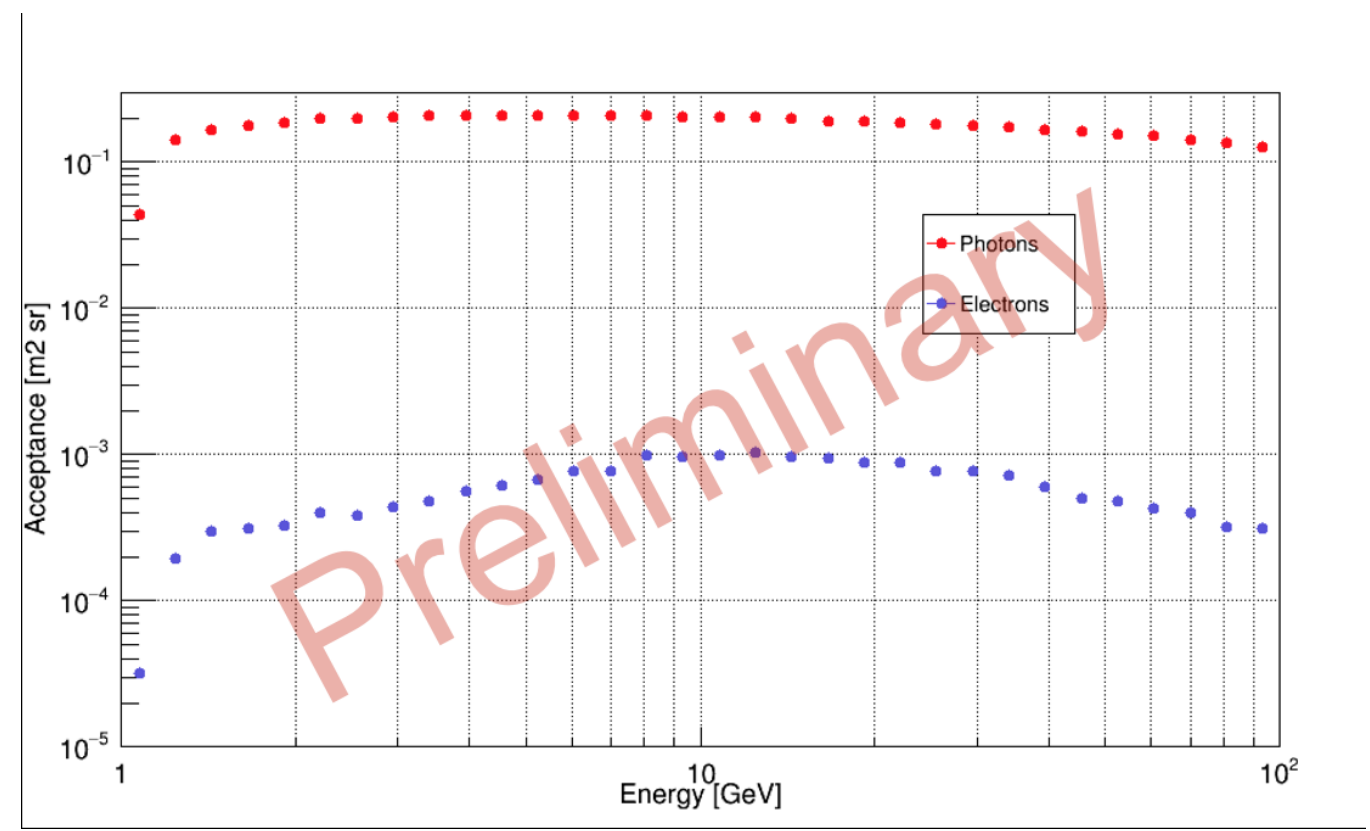

Figure 1: Acceptance of gamma-rays and electrons for the gamma-ray selection applied for the present analysis. From $1 \mathrm{GeV}$ to $100 \mathrm{GeV}$ in logarithmic scale.

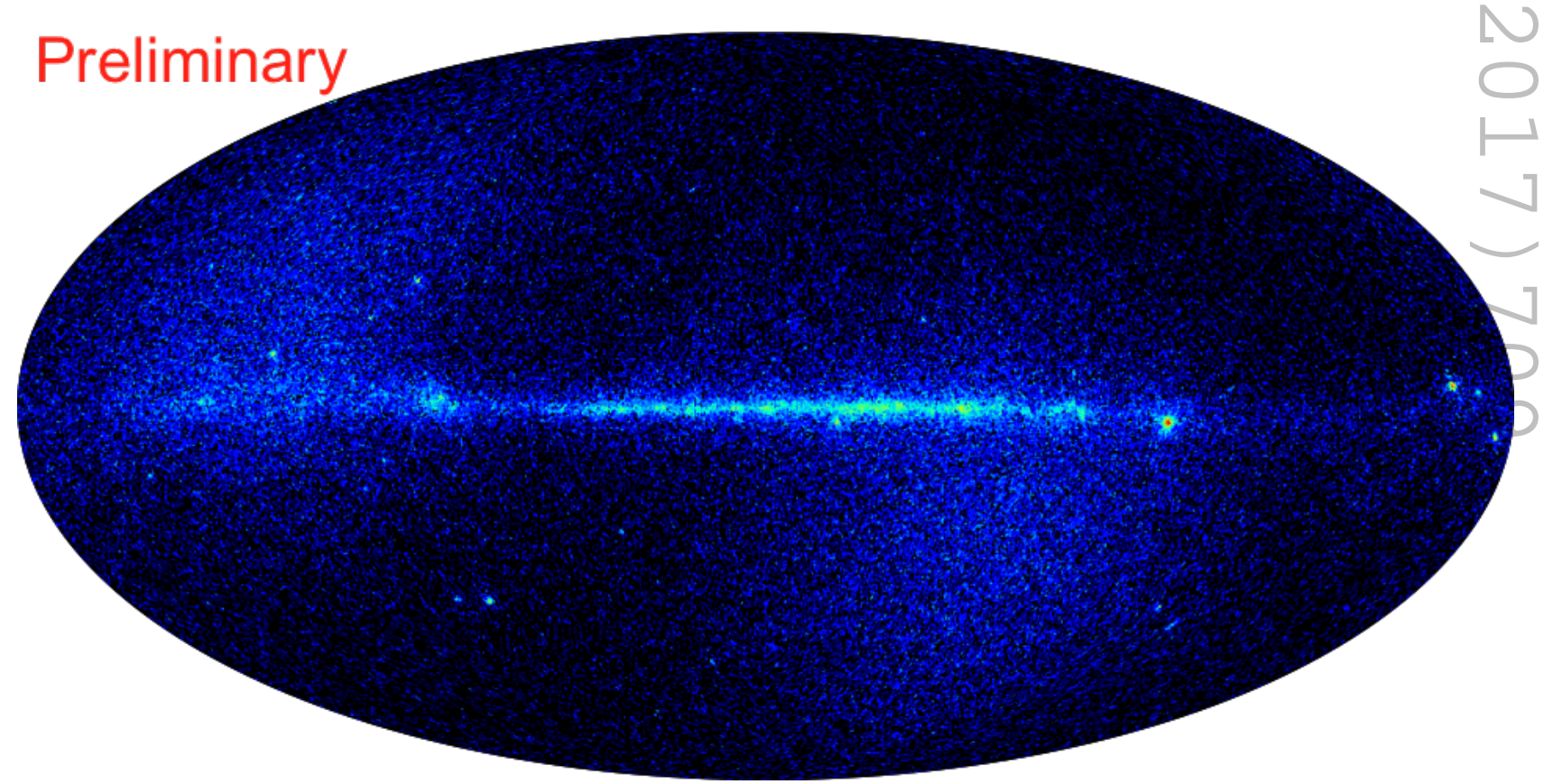

Figure 2: Preliminary skymap for 18 months of the DAMPE on-orbit measurements in survey mode. It is presented in a Mollweide projection in Galactic coordinates. The plotted photon candidates, have raw energies from $1 \mathrm{GeV}$ to $100 \mathrm{GeV}$

\section{Pulsar Analysis}

Since pulsars were discovered in 1967 by Jocelyn Bell and Anthony Hewish they have been a 
source of great interest. Pulsars are highly-magnetized rapidly rotating neutron stars formed during the collapse of a massive star into its core after it runs out of nuclear power. The main characteristics of a pulsar are their period and their first derivative of this period (spin-down rate).According to those a pulsar can be classified from millisecond pulsars (MMPs) to young pulsars. Pulsars can also be grouped in radio-quiet or radio-loud pulsars depending on the energy of their emission. The timing solutions of pulsars can be measured from earth by dedicated experiments in radio and X-ray. Space-born experiments like EGRET or FERMI opened the window to more precise measurements at $\mathrm{GeV}$. For studies at very higher energies results have been shown with on-earth Cherenkov telescopes such as MAGIC or HAWK. The knowledge on the pulsar parameters can be used to produce the pulsation profiles which in turn allow us to make a clear distinction between photons, which come form the pulsed component, and background photons or electrons which induce a flat distribution in the light curve.

For being able to compute the phase we use the pulsar timing solutions (ephemeris) of interest in combination with the time-stamp and position recorded for each event, and the known position in the sky for the different sources, normally measured by other experiments. In this case all the ephemeris were provided by David A. Smith (Private Communication, 2017).

Times obtained from DAMPE are given in the Mission Elapsed Time (MET) TT, which corresponds to the number of seconds elapsed since the 1st of January 2014 at 00:00 (UTC). The MET, which is calculated from the timing from a GPS receiver on board is used to assign a time-stamp to each event. The position of the satellite with respect to the Earth's center, based on GPS information, is stored for each event as well. Pulsar timing analysis software, was created at the university of Geneva to be compatible with the format of the DAMPE data. This software is based in Open Source Code to transfer times into different frames of interest using the MET and the information on the position of DAMPE taken from the housekeeping data. The program converts the MET time to the topocentric time by using the position of the satellite, then a conversion from the topocentric to the barycentre of the solar system is performed, as required to fold the light curve of a pulsar. Meaning that we apply correction on the effects of DAMPE and the Earth's orbit respectively. Since the barycentre depends on the position of the source we are using as reference, and different sources may have changes in their position we correct also for this. Each object in the sky will vary at different rates with respect to the solar system, this variation is called Proper Motion (PM). For objects in the sky with known proper motion this value was considered and corrected for each event. The variation in position occurs as function time therefore we use the MET before time frame related corrections.

Once times are in the solar system barycentre frame, they can be folded into the phase. For this we require to define an epoch $E_{0}$, that is the epoch of reference that defines the beginning of the phase. The equation 2.1

$$
P=f \times t_{i}+\frac{f_{1} \times t_{i}^{2}}{2}+\frac{f_{2} \times t_{i}^{3}}{6}
$$

Where $t_{i}=E_{0}-t_{\text {bary }}, t_{\text {bary }}$ the time in the barycentre of the solar system, $f, f_{1}, f_{2}$ are the frequency, its first and second derivative respectively and $P$ the calculated phase.The resulting value is the number of periods that have occurred since the epoch of reference $E_{0}$. The value we are interested in is the fraction, since this tell us the pulsar phase for the given photon candidate. 
For each source we select events within $3^{\circ}$ of the pulsar known position. Due to the orbit of the DAMPE satellite we have a non-uniform sky exposure pulsars are observed during different periods of time. The data is divided into different periods and the ephemerides, including information on the glitches, received from David A. Smith for these periods were used.

\subsection{Geminga Pulsar}

The second brightest non-variable source in the gamma-ray sky. Geminga was the first radioquite pulsar to be observed. It is an old pulsar and therefore it has a very stable first period derivative. Geminga is one of the objects in the sky with the fastest proper motion (PM) measured. Correction in the position as function of time is applied, for each event. Geminga has been one of the most studied pulsars in the last years. Multi-wavelength studies are one of the main target for this pulsar, due to the emission at very high energies $(>100 \mathrm{GeV})$.

Preliminary results show the light curve of the Geminga pulsar with its characteristic double peaked phase and a separation of $\sim 0.5$ phase in Figure 3. The data used was selected from the first year of operation of DAMPE from the 1st of February 2016 to the 21st of May 2017, obtaining 682 events. Highest energy events have been measured up to $\sim 50 \mathrm{GeV}$.

Results match the with previous results showed by EGRET, FERMI, among others [5] [3].

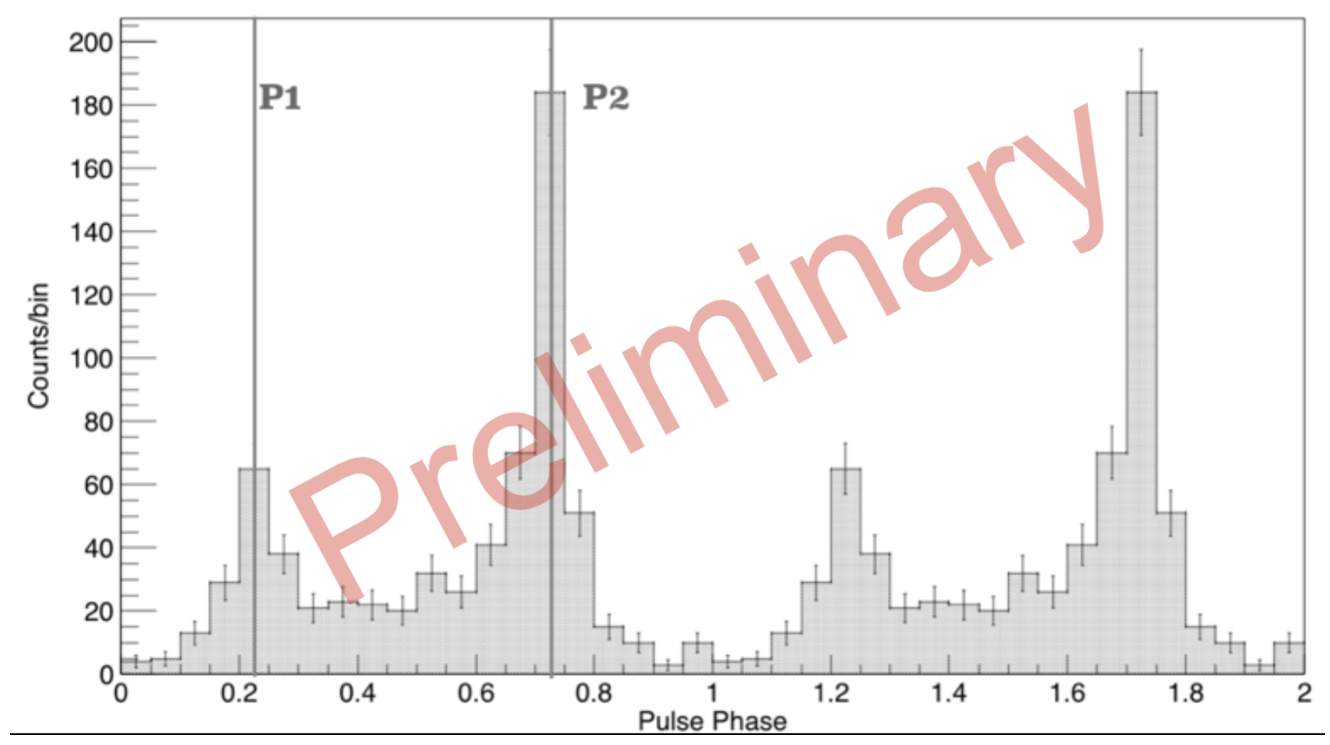

Figure 3: Geminga light curve for energies above $1 \mathrm{GeV}$. Two pulse phase periods are presented with a precision of 20 bins per period.

\subsection{Vela Pulsar}

Vela is the brightest non-variable source in the gamma-ray sky. The Vela pulsar emits as well in VHE $>100 \mathrm{GeV}$ From the 1st of January 2016 to the 20th of November 2016, DAMPE has recorded 1004 events coming from Vela's direction within a ROI of $3^{\circ}$. The highest energy events recorded, has an energy of $\sim 90 \mathrm{GeV}$ the Vela pulsar has period of $\sim 89 \mathrm{~ms}$, its characteristic light curve is plotted in figure 4 with a precision of 20 bins per phase, $\sim 4.45 \mathrm{~ms}$ per bin. Since Vela is 
the brightest source, it also has the most statistics of the present study, showing that by increasing the statistics, there is a better agreement with the light curves described by other experiments. In figure 4 we can observe the light curve a three peak structure in our energy range. Below $1 \mathrm{GeV}$ the pulsar is known to have a two peak structure, with the third peak more primient at higher energies.[2]

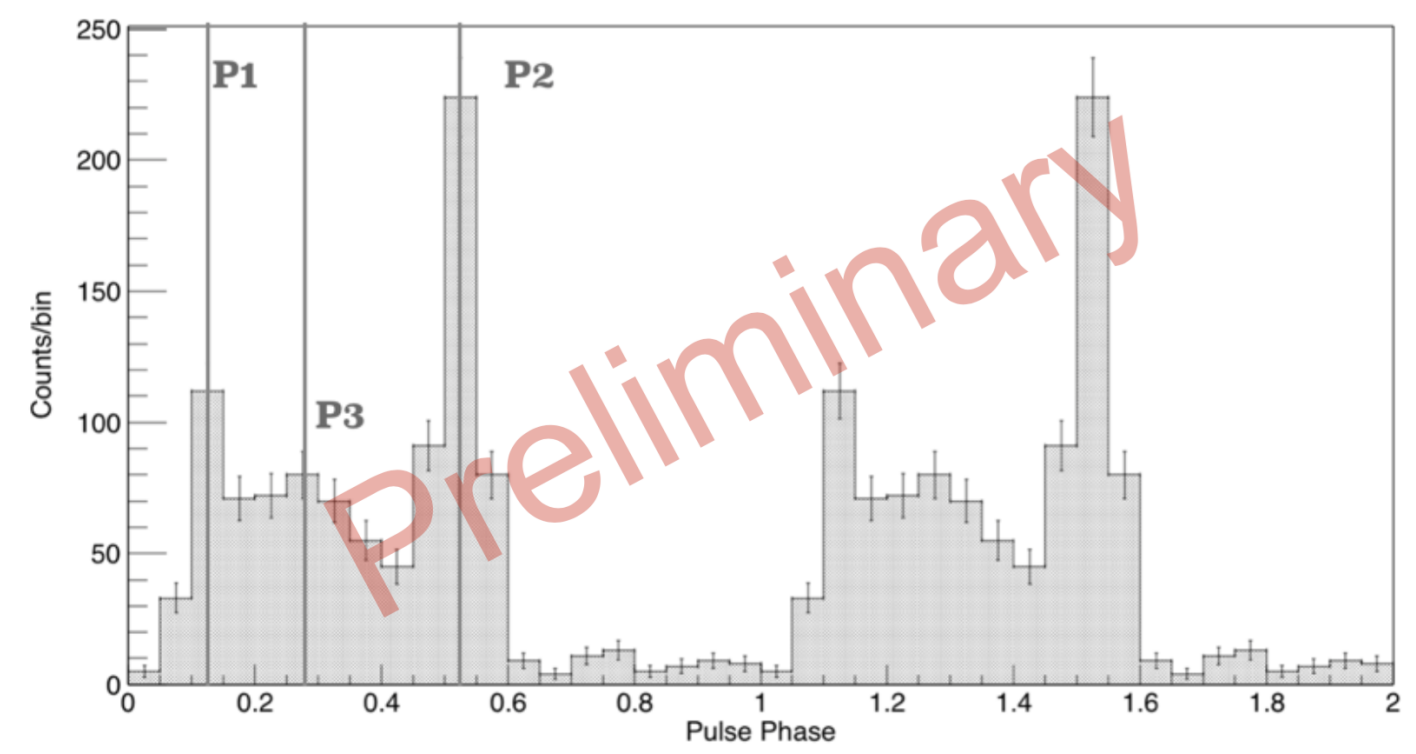

Figure 4: Vela light curve for energies above $1 \mathrm{GeV}$. Two pulse phase periods are presented with a precision of 20 bins per period.

\subsection{PSR J0007+7303}

Pulsar J0007+7303 was first detected by Fermi-LAT [4], associated to the supernova remnant CTA1. After its first detection and timing solutions coming from Fermi-LAT, afterwards a multi-wavelength analysis took place amont different experiments, XMN-NEWTON uncovered the X-ray counterpart. Further studies for the search of optical and radio signal did not show any result. Making J0007+7303 a radio-quiet pulsar. Observations performed since 2004 by Chandra determined that J0007+7303 is Pulsar Wind Nebula (PWN) [6].

DAMPE has observed this object from the 1st of January 2016 to the 31st of January 2017, detecting 141 photon candidates, from this events the highest energies recorded is $\sim 25 \mathrm{GeV}$. In Figure 5, we can observe the resulting light curve for these period of observation using a timing solution. J0007+7303 has a period of $\sim 316 \mathrm{~ms}$. The light curve is plotted with 24 bins per phase, that represent $\sim 13.16 \mathrm{~ms}$ per bin. The profile shows two peaks separated $\sim 0.2$ of a phase. Results from fermi show a separation to be 0.180 [4].

\section{Conclusions and Future Scope}

Using data from the first 18 months during which the DAMPE satellite has been in orbit we have shown it to be an excellent instrument for the identification and analysis of pulsars in an energy 


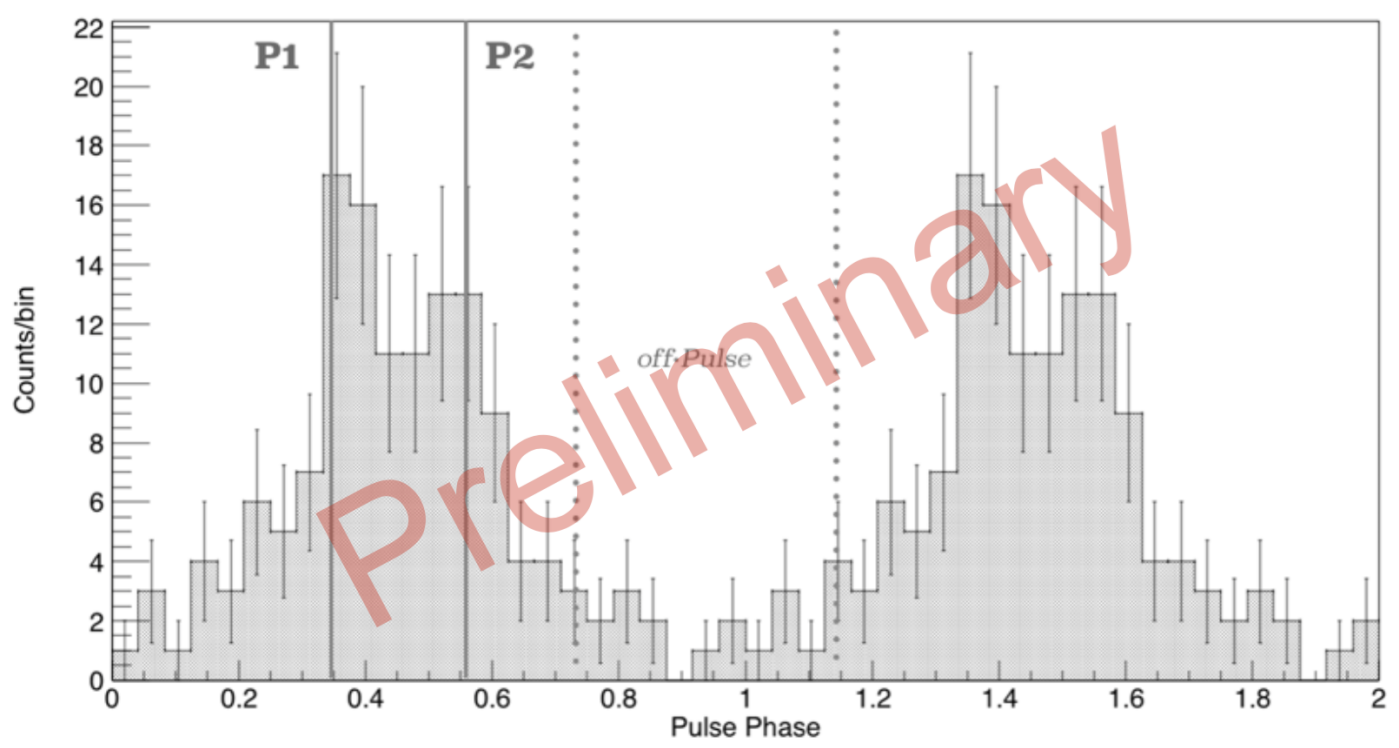

Figure 5: J0007+7303 light curve for energies above $1 \mathrm{GeV}$. Two pulse phase periods are presented with a precision of 24 bins per period.

range from 1-100 GeV. Future work will focus on further improving the photon event selection and acquiring more statistics, allowing us to investigate the pulsation of these and potentially more pulsars for energies above $100 \mathrm{GeV}$. We can then perform spectral analysis in the pulse and offpulse regions, observe lights curves evolutions as a function of energy. We also have shown the broad possibilities for the search an study of gamma-ray sources such as the galactic plane, SNRs, and AGNs among others. The objective of DAMPE which has an excellent energy resolution in the $1-100 \mathrm{GeV}$ energy range, is to provide detailed energy measurements in the $1-100 \mathrm{GeV}$ energy range during the following years of the campaign. Our current short term objective is to confirm the capabilities of DAMPE to perform pulsar studies and use the results to improve our event selection.

\section{Acknowledgments}

MMS expresses her gratitude to David A. Smith for providing the ephemeris applied for this analysis and valuable advise as well as Nicolas Produit for the first introduction towards pulsars analysis and timing corrections. MMS also acknowledges the financial support from the ICRC committee to attend the meeting.

The DAMPE mission is funded by the strategic priority science and technology projects in space science of Chinese Academy of Sciences. In China the data analysis was supported in part by the National Key Research and Development Program of China (No. 2016YFA0400200), National Basic Research Program of China (No. 2013CB837000), NSFC (Nos. 11525313 and 11622327), and the 100 Talents Program of Chinese Academy of Sciences. In Europe the activities and the data analysis are supported by the Swiss National Science Foundation (SNSF), Switzerland; the National Institute for Nuclear Physics (INFN), Italy. 


\section{References}

[1] J. Chang et al. [DAMPE Collaboration], "The DArk Matter Particle Explorer mission," arXiv:1706.08453 [astro-ph.IM].

[2] A. A. Abdo et al. [Fermi-LAT Collaboration], "Fermi LAT Observations of the Vela Pulsar," Astrophys. J. 696, 1084 (2009) [arXiv:0812.2960 [astro-ph]].

[3] A. A. Abdo et al. [Fermi-LAT Collaboration], "Fermi LAT observations of the Geminga pulsar," Astrophys. J. 720, 272 (2010) [arXiv:1007.1142 [astro-ph.HE]].

[4] A. A. Abdo et al., "PSR J0007+7303 in the CTA1 SNR: New Gamma-ray Results from Two Years of Fermi-LAT Observations,” Astrophys. J. 744, 146 (2012) [arXiv:1107.4151 [astro-ph.HE]].

[5] A. A. Abdo et al. [Fermi-LAT Collaboration], "The Second Fermi Large Area Telescope Catalog of Gamma-ray Pulsars,” Astrophys. J. Suppl. 208, 17 (2013) [arXiv:1305.4385 [astro-ph.HE]]

[6] J. P. Halpern, E. V. Gotthelf, F. Camilo, D. J. Helfand and S. M. Ransom, "X-ray, radio, and optical observations of the putative pulsar in the supernova remnant CTA 1," Astrophys. J. 612, 398 (2004) [astro-ph/0404312]. 